\title{
Fluid features of the stochastic layer transport in LHD
}

\author{
Y. Feng, M. Kobayashi, T. Morisaki, S. Masuzaki, J. Miyazawa,
} B.J. Peterson, S. Morita, M. Shoji, K. Ida, I. Yamada, K. Narihara, N. Ashikawa, H. Yamada, N. Ohyabu, A. Komori, O. Motojima and the LHD experimental group, F. Sardei , D. Reiter

\author{
National Institute for Fusion Science, Toki, Japan \\ Max-Planck-Institut für Plasmaphysik, Euratom Association, Greifswald, Germany \\ Institut für Plasmaphysik, Forschungszentrum Jülich Gmbh, Euratom Association, Trilateral \\ Euregio Cluster, D-52425 Jülich, Germany
}

\begin{abstract}
The stochastic scrape-off layer (SOL) of the helical divertor configuration in LHD exhibits a rather complex field topology where remnant magnetic islands, thin edge surfaces and stochastic field lines coexist. Using the three-dimensional (3D) edge transport code package, EMC3EIRENE, the paper presents a numerical study of the stochastic layer transport, aimed, first of all, at clarifying to what extent the plasma as a fluid is influenced by the stochastic behavior of the magnetic field lines. Revealed and analyzed are the individual roles of different regions of the stochastic layer for plasma, impurity and neutral transport. Topics addressed and discussed are island flattening effects, flow patterns and flow damping, recycling flux suppression, rollover of divertor flux, detachment and Marfe formation and dynamics. Comparisons with experimental results are also presented.
\end{abstract}




\section{Introduction}

Associated with the large shear, the rotational transform in the edge region of the LHD helical divertor (HD) configuration [1] covers countless resonances which overlap each other, forming a stochastic layer of $\sim 10 \mathrm{~cm}$ thickness surrounding the confinement core region. The stochastic layer provides an intermediate zone between the confinement core and the plasma-surface interaction region, screening the confinement core from direct penetration of recycling neutrals and sputtered impurities. Hence, the intermediate stochastic SOL is expected, to a large extent, to affect the performance of the HD, especially for the present open divertor configurations. Unlike the standard SOL in tokamaks and the closed or nearly closed island SOLs in the low-shear stellarators W7-AS [2] and W7-X [3], the stochastic SOL in LHD exhibits a complex field structure characterized by coexistence of remnant magnetic islands, stochastic fields and edge surface layers. Deep in the SOL, the moderate shear does not destroy the low-order islands completely, so that closed island surfaces still exist in limited regions around the O-points. Moving outwards, the shear increases quickly, leading to stronger island overlap. The remnant island cores become smaller and eventually disappear. In this case, the Chirikov parameter [4] should largely exceed unity and the field lines are then expected to behave diffusively [5]. In reality, however, thin open surfaces have been identified to exist within the outer, strong islandoverlapping region in LHD [1]. The existence of such thin surface layers is further confirmed by connection length contour plots which show clearly thin channels filled with rather long field lines connecting zones of completely different connection lengths. In fact, the four divertor legs, on which the HD is based, result from such thin surfaces. This strong field-line correlation contradicts obviously the diffusive behavior of stochastic field lines. In the inner region adjacent to the thin surface layers, open field lines and closed remnant island cores coexist. Poincare-plots 
do show some irregular patterns of the open field lines surrounding the closed island cores, however, they cannot provide any useful information about the stochasticity.

In view of the large differences in field and divertor geometry between W7-AS and LHD, it is interesting to see whether there exist certain common physics issues in terms of divertor transport or divertor functionality. Recently a collaboration work between IPP and NIFS was started for this purpose and the paper presents the first application results of the EMC3[6]-EIRENE[7] code for the HD in LHD, taking self-consistently the plasma, neutral and the intrinsic-carbon impurity transport into account. Topics addressed are particle flux enhancement, neutral screening, impurity radiation and detachment, as already extensively studied for the W7-AS island divertor (ID) (see e.g [8]). They are also the important basic elements of a divertor which need to be firstly investigated and understood especially for a divertor concept based on a complex 3D field structure like the ID in W7-AS and the HD in LHD. The paper is, in particular, concentrated on understanding the elementary, global transport processes associated with the specific field topologies, aiming at forming a comparison basis for two typical helical devices of completely different divertor concept and geometry. Effects related to plasma currents and drifts widelydiscussed in tokamaks (see e.g. $[9,10]$ ) are not addressed in this paper. Their relevance in individual helical devices is being examined and a quantitative assessment of the drift effects in 3D helical SOLs is left for an upgraded 3D code version after implementation of the corresponding drift terms.

\section{Computational set-up}

The EMC3 code solves a set of stationary standard fluid equations in a real 3D space of arbitrary magnetic field geometry using an advanced Monte Carlo technique. Fluid transport is 
approximated by a stochastic process using three local, orthogonal, field-aligned basis vectors. In order to realize a clean separation between the highly anisotropic parallel and perpendicular transport, the fast parallel transport process is integrated over a finite field-line length [11]. For this, field lines are needed in the whole computational domain. In order to avoid a timeconsuming field-line integration procedure for individual Monte-Carlo particle trajectories, the reversible field line mapping technique, RFLM [12], is adopted in the 3D code to interpolate field lines from pre-calculated ones stored on a given mesh. Therefore, the computational mesh in the 3D code has two functionalities, i.e. field line interpolation and plasma parameter representation.

A computational mesh is constructed for a vacuum configuration with $\mathrm{R}_{\mathrm{ax}}$ (magnetic axis $)=3.75 \mathrm{~m}$. This is considered to be a reasonable approximation for the low $-\S(<\beta><0.5 \%)$ discharges (full field, $\mathrm{P}_{\mathrm{NBI}} \sim 8 \mathrm{MW}, \bar{n}<8 \times 10^{19} \mathrm{~m}^{-3}$ ) investigated in the paper. In view of the stellarator symmetry, only a half field period is taken into account. In order to avoid a strong mesh deformation violating the reversibility of the interpolating scheme adopted in the RFLM technique, the computational domain has been divided into two toroidal sections with the interface being located in the middle of the toroidal domain. The two mesh sets are constructed in a similar way. Figure 1 a) shows the cross-section of a subset of one mesh on an up/down symmetric plane. The mesh starts radially from a deep core region which the recycling neutrals can reach and terminates at the wall, covering the core, SOL and vacuum regions. The core region is introduced only for neutral transport, with the plasma being pre-defined according to profile measurements. The innermost radial boundary is a flux surface which is shared by the two mesh sets to ensure a closure of the whole computational system at the innermost boundary. Plasma transport is simulated only in the SOL region enclosed between the last closed flux surface (LCFS) and an outer surface determined after careful examination of the field topology 
and properties, as discussed later in some detail. The four divertor legs indicated by the dashed lines in figure 1 a) are not included explicitly in the computations for technical reasons. Hence, a plasma vacuum region is assumed to exist between the SOL and the wall. This simplification should not influence the presented results significantly because a) the divertor legs are optically too thin to stop the recycling neutrals, as indicated by $\mathrm{H}_{\alpha}$-measurements, b) the connection length is too short to cause remarkable parallel plasma gradient [13] and c) our studies are focused on the transport in the stochastic layer.

Because of the absence of the divertor legs in the mesh, equivalent parallel plasma loss channels have to be defined. This is realized by careful choice of the outermost boundary surface in the SOL. Instead of going into technical details of the surface determination process, we describe the basic properties of such a surface, as shown in figure 1 a) and b). The outermost surface, which has a smooth, up/down symmetric form on the symmetric plane shown in figure 1a), becomes deformed after forward mapping along field lines over $1 / 4$-field period up to the interface, with two tails being formed around the tips of the ellipse, as indicated by A and A' in figure $1 \mathrm{~b}$ ). Similarly, two other tails B and B' are found for the second toroidal section of the mesh after backward mapping of the outermost surface over $1 / 4$-field period up to the interface. The four tails indicate the positions where field lines leave the stochastic layer region for the targets. In fact, they form the initial parts of the neglected divertor legs. Therefore, the surface areas of the four tails are treated as virtual plates for plasma transport in the simulations. This is a reasonable approximation because of the rather short connection lengths of the real divertor legs [13]. For determining the neutral source distribution, however, the ion fluxes through the virtual plates are mapped onto the real targets and the virtual plates are not seen by the neutrals.

\section{Plasma transport properties prior to detachment}




\subsection{Island effects}

Figure 2 shows the connection length contours of field lines in the edge region of the vacuum field configuration for $\mathrm{R}_{\mathrm{ax}}=3.75 \mathrm{~m}$. In order to reveal finer magnetic structures, the contour plot is zoomed in radial direction as shown in the lower picture of figure 2, with a Poincare plot overlying. Because of the large shear in the edge, the rotational transform increases from $\sim 1$ to $\sim 5$ in the region investigated, covering a large number of resonances. The low-order island chains with the poloidal mode numbers from 5 to 8 can be easily identified. The large shear compresses the low-order islands within a thin layer $(\sim 10 \mathrm{~cm})$, leading to island overlapping. This happens already for the 10/7 island chain where the island width is evidently comparable with the distance to the 10/6 island chain. In this case, the Chirikov parameter approaches unity and the field becomes stochastic. This is, indeed, shown by the overlying Poincare plot where most of the field lines in the radial range of the 10/7 island chain become irregular, with only small island cores remaining around the O-points. If one, however, looks at the underlying $\mathrm{L}_{\mathrm{c}}$-contour carefully, one finds that the basic form of the island chain still remains, meaning that the field lines within the islands correlate strongly in spite of the stochastic behavior indicated by the Poincare plot. Moving outwards, both the growing island size and the increasing shear should make the field more stochastic because of the resulting, rapidly-increasing Chirikov number. This is reflected by the vanishing of the remnant island cores from the $\mathrm{m}=2,3,4$ and 5 mode structures. Topologically and magnetically, it is difficult to understand how the island cores of these lowest-

order mode structures are suddenly opened, with, however, much longer field lines surrounding them. The long field lines form edge surfaces, which is a peculiar feature of the LHD configuration [1]. The existence of the edge surfaces and the fact that most of the low-order mode structures are still identifiable from the $\mathrm{L}_{\mathrm{c}}$-contours indicate that the basic magnetic structures of 
the low-order modes are not completely destroyed and the field lines seem to be more 'regular' than stochastic.

EMC3/EIRENE simulations have been made in order to check how and to what extent the plasma as a fluid follows the complex field structures shown above. Hydrogen plasma is assumed. The power entering the SOL is set to be $8 \mathrm{MW}$. Cross-field transport coefficients are determined by comparison with Thomson profiles, which will be shown later. They are co-varied as $\chi_{\mathrm{e}}=\chi_{\mathrm{i}}=3 \mathrm{D}$ and are kept spatially constant (global transport). Figure 3 shows the resulting $\mathrm{T}_{\mathrm{e}^{-}}$ contour and parallel ion flow distribution. Poincare plots are overlaid in order to show the correlation between the plasma distributions and the island geometry. In contrast to the $\mathrm{L}_{\mathrm{c}^{-}}$ contour shown in figure 2, the calculated $\mathrm{T}_{\mathrm{e}}$ exhibits a much smoother distribution because of the smoothing effect of the perpendicular transport. Energy entering a magnetic island through the inner separatrix is guided by the field lines through fast parallel transport outwards to the island tip and leaves the island again through perpendicular transport. This process is schematically sketched in figure 4 where a regular island is shown and the stochastic effect is completely ignored. Because of the high classical electron heat conductivity, the parallel heat conduction tends to smooth $T_{e}$ throughout the island chains. This can be clearly seen in figure 3 for the 10/8, 10/7 and 10/6 islands. Even for the 10/7 and 10/6 islands, which have a large surrounding stochastic region, the underlying island form emerges clearly in the $\mathrm{T}_{\mathrm{e}}$-contour, without any evidence of effects resulting from a random trajectories of field lines. Associated with the finite classical heat conductivity, the parallel conductive heat flux in the islands implies a parallel temperature drop from the inner separatrix to the island tips, especially for ions due to their weaker parallel heat conductivity. The parallel temperature gradient in the momentum balance acts to drive a parallel plasma flow. The principle is illustrated in figure 4 where positive and 
negative flows reside on different sides of the island due to the stagnation at the upstream position of the inner separatrix. The parallel plasma flows calculated by the 3D code are shown in the bottom picture in figure 3 where positive and negative flows surround the O-points, with the flow patterns clearly correlating with the island structure. Even for the low mode number "islands" without a closed island core, flow channels residing on the island chains are still identifiable. Thus, we can conclude that the plasma transport in the stochastic layer is governed by the low-order islands, although we could not yet quantify the relative importance of the stochastic effects with respect to those of regular islands.

\subsection{Comparison with experiments}

Figure 5 compares the electron temperatures between simulations and Thomson scattering measurements along the midplane on a symmetric cross-section with the long axis lying horizontally, as shown in the upper picture in figure 2. The laser passes through the SOL region two times and the profiles on both the in- and out-board sides are shown. The simulation results are obtained with $\mathrm{D}=0.5 \mathrm{~m}^{2} / \mathrm{s}$ and $\chi_{\mathrm{e}}=\chi_{\mathrm{i}}=3 \mathrm{D}=1.5 \mathrm{~m}^{2} / \mathrm{s}$. Note all the perpendicular transport coefficients are spatially constant. A sensitivity study of the numerical results on the cross-field transport coefficients can be seen in the next section. On the inboard side, the 10/3, 10/5 and 10/7 “islands” reside just on the midplane (see figure 2) and therefore appear in the $\mathrm{T}_{\mathrm{e}}$-profiles of both the simulations and measurements. The flattening effect of the 10/7 island chain, which is already shown in figure 3, now becomes clearer in figure 5. The 10/6 island chain has a poloidally-shifted phase distribution, with an X-point being located on the inboard midplane. That is the reason why it does not appear in the Te-profiles. On the way to the $10 / 5$ island, $\mathrm{T}_{\mathrm{e}}$ drops strongly because the cross-field heat conduction dominates the energy transport between the neighboring island chains. Then, $\mathrm{T}_{\mathrm{e}}$ reaches a minimum around the O-point of the 10/5 island due to the stronger energy 
sink there associated with the shorter connection lengths with respect to the surrounding areas around the separatrix (see figure 2). The long connection length stripe forms a parallel power channel transporting energy from the inner separatrix to the island tip. This explains why $T_{e}$ recovers slightly on the way from the O-point outwards to the island tip, this being a typical 2D or 3D effect. A similar phenomenon occurs also for the $10 / 3$ island and the $\mathrm{T}_{\mathrm{e}}$-peak at the island tip becomes now more pronounced. The $T_{\mathrm{e}}$-peak position is simply associated with the thin power channel formed by the edge surfaces which is clearly shown in figure 2. On the outboard side, all the low-order "islands" have their X-points on the midplane, which is the reason for the much smoother $\mathrm{T}_{\mathrm{e}}$-profiles there. The measured and calculated $\mathrm{T}_{\mathrm{e}}$-profiles agree well in the outer region starting from the 10/7 islands. Nevertheless, systematic deviations occur in the inner region where the simulated $\mathrm{T}_{\mathrm{e}}$-slopes are much flatter than the measured ones. In order to approach the steep $\mathrm{T}_{\mathrm{e}}$-gradient measured in the inner region, the perpendicular transport coefficients in the simulations have to be reduced roughly by a factor of 3 . This leads to a significant rise of $T_{e}$ in the outer region, with the simulated $T_{e}$-profile being clearly above the measured one. Besides the directly-reduced cross-field conductive heat flux, there are two effects which could contribute to the increase in $T_{e}$ indirectly. Firstly, the parallel energy transport channels become narrower with decreasing perpendicular transport, resulting in a higher parallel heat flux density. Secondly, when the parallel heat channels shrink towards the island separatrix, the effective connection length will increase due to the singularity of X-points. Simulations using overall constant cross-field transport coefficients cannot reproduce the measured $\mathrm{T}_{\mathrm{e}}$-profiles in the inner and outer regions simultaneously. This suggests a change of the plasma transport properties around the interface between the 10/7 and 10/8 island chains, thus indicating the boundary between the SOL and the core. 


\subsection{Absence of a high recycling regime}

The linear and nonlinear SOL regimes found in tokamaks are examined for the helical divertor configuration discussed here. In experiments, a density ramp is carried out for hydrogen plasmas with a fixed NBI-heating power of $\sim 8 \mathrm{MW}$. Plasma density is varied in a reasonable range to cover the conditions for different SOL transport regimes. In simulations, the power into the SOL, $\mathrm{P}_{\text {SoL }}$, is fixed to be $8 \mathrm{MW}$ without corrections related to the deposited power and the core radiation. This is a reasonable assumption when the line-average density is higher than $3 \times 10^{19} / \mathrm{m}^{-}$ ${ }^{3}$. At lower densities, the deposited power decreases because of the shine-through particles. Within the density range investigated, no significant core radiation is detected [14]. The lineaveraged density $\overline{\mathrm{n}}$ used as a control parameter in the density ramp experiments is not an input parameter for the 3D code. Instead, the plasma density on the LCFS, $\mathrm{n}_{\mathrm{LCFS}}$, as a boundary condition, is varied in the simulations. A direct comparison with the experiments needs therefore the relationship between $n_{\text {LCFS }}$ and $\bar{n}$. This relationship is experimentally determined by the microwave interferometer and Thomson scattering measurements. It is found that $\mathrm{n}_{\mathrm{LCFS}} \approx 0.8 \overline{\mathrm{n}}$. Furthermore, in order to check the sensitivity of the simulation results to the cross-field coefficients, two different sets of D are selected, $\mathrm{D} \equiv 0.5 \mathrm{~m}^{2} / \mathrm{s}$ and $\mathrm{D} \sim 1 / \overline{\mathrm{n}}$.

The numerical and experimental results are compared in figure 6 , using $\overline{\mathrm{n}}$ as an independent parameter. The upper figure shows the $\bar{n}$-dependences of the calculated total recycling flux (total ion outflow onto targets) and of the ion saturation currents integrated over the target Langmuir probes at a toroidal location of maximum recycling. The ion saturation currents measured by the Langmuir probes increase linearly with increasing $\overline{\mathrm{n}}$ up to a rollover of $I_{\text {sat }}$ around $\overline{\mathrm{n}}=7.5 \times 10^{19} / \mathrm{m}^{-3}$. There is no evidence of transition to a high recycling regime as usually observed in the tokamak poloidal-field divertor. The linear $\mathrm{I}_{\text {sat }}$-behavior can be well 
reproduced by the simulations, independent of the applied D-ansatz. Nevertheless, the use of $\mathrm{D}=0.5 \mathrm{~m}^{2} / \mathrm{s}$ results in a recycling flux slope identical to that of $\mathrm{I}_{\mathrm{sat}}$, whereas the $1 / \mathrm{n}$-scaling provides a much flatter one, indicating a density-independence of the anomalous transport for the NBI-discharges investigated. This is further supported by the upstream $T_{e}$ comparison between the simulations and measurements, as shown in the lower part of figure 6 . In the density range of $\overline{\mathrm{n}}>3 \times 10^{19} / \mathrm{m}^{-3}$, the Thomson channels viewing upstream show a remarkable, monotone decrease of $T_{e}$ with increasing $\bar{n}$. In the simulations, a comparably strong $T_{e}$-drop can only reproduced by keeping D constant throughout the density ramp. In contrast, when D scales inversely with $\mathrm{n}$, the upstream $\mathrm{T}_{\mathrm{e}}$ remains almost unchanged. The systematic departure of the calculated temperatures with the constant $\mathrm{D}$ from the measured ones in the lower $\overline{\mathrm{n}}$-range is considered to result from a reduced NBI-heating efficiency, as already mentioned at the beginning of this section. This will be proved by NBI power deposition calculations.

The absence of a high recycling regime in the LHD helical divertor configuration investigated is understood as a consequence of momentum loss resulting from the friction between the counter flows, as already found in the W7-AS island divertor [8]. As shown in figure 3 and already discussed in section 3.1, parallel plasma flow patterns correlate closely with the island structure, i.e. opposite flows reside on neighboring island fans. Then, viscous momentum transport can cause significant momentum losses when positive and negative flows approach each other. The frictional flow interaction occurs mainly in the radial direction because of the small radial separation of the island chains and is intensive especially at poloidal positions where the neighboring island chains have an appropriate phase distribution, e. g. around the midplane on the inboard side shown in figure 3. The geometry-related momentum losses break up the parallel momentum conservation and thereby suppress a high recycling regime. 


\subsection{Rollover of the divertor recycling flux}

As shown in figure 6, the ion saturation current drops when the plasma density is increased beyond a critical value. The rollover effect can well be reproduced by the 3D code, with the predicted rollover point of density for the $\mathrm{D}=$ const case being in good agreement with the measured one. A drop of the total recycling flux (the total ion outflow) means a reduction of the thermal power diverted onto the targets, provided the downstream $T_{e}$ drops monotonically with increasing $n_{\mathrm{e}}$. This implies that, for a given amount of SOL power, an additional power loss channel exists in the SOL. Indeed, the residual power fraction is radiated by impurities. In the simulations, carbon impurities released from the graphite targets have been taken into account. Around the rollover point, chemical sputtering is considered to be the major carbon impurity production process. The carbon release flux is linearly coupled to the hydrogen ion flux on the target, with the sputtering coefficient (the ratio of the carbon-release to the hydrogen ion flux) being introduced as a free input parameter in view of the large uncertainties existing in wallconditioning. $2 \%$ carbon sputtering is assumed in the presented calculations. Certainly, the threshold density for the onset of rollover depends on the sputtering coefficient selected. Nevertheless, a high sensitivity is not expected [8]. In addition, our attention is focused on understanding the physics behind the rollover effect, rather than on determining an absolute carbon yield by fitting the measured rollover point iteratively. As will be shown later in the next section, the carbon radiation increases significantly when the recycling flux drops.

Additional power removal e. g. through impurity radiation is a precondition, but not the only one for decreasing the particle flux onto the target plates. The particle flux is a quantity of particle transport governed by momentum balance. A direct consequence of impurity radiation is to lower the downstream temperature. This in turn tends, under the condition of parallel 
momentum conservation, to enhance the recycling flux, causing a thermal instability, as already known from tokamaks [15]. Thus, the linear coupling between the upstream and downstream pressures has to be broken up. In tokamaks, this is realized under low- $T_{e}$ conditions where the parallel momentum of the streaming ions is removed by the recycling neutrals through chargeexchange processes [16]. As already discussed in the previous section, the overlapping island chains in LHD cause significant momentum losses between the counter flows by means of friction, accounting for the absence of a high recycling regime. However, a reduction of the recycling flux needs an enhancement of the momentum loss. This is considered to be caused by the inward shift of the ionization front. Figure 7 shows the radial profiles of the ionization sources for three density cases around the rollover point with $\mathrm{n}_{\mathrm{LCFS}}=5,6$ and $6.4 \times 10^{19} \mathrm{~m}^{-3}$, respectively. Their locations on the $\overline{\mathrm{n}}$-axis in figure 6 can be found using the relation $\mathrm{n}_{\mathrm{LCFS}}=0.8 \overline{\mathrm{n}}$. The ionization sources are averaged over the poloidal and toroidal computational cells. The 'effective' radius is defined by cylindrical approximation of the volumes enclosed by each radial surface in the computational mesh. At densities prior to the rollover point, most of the ionization takes place in the edge surface layer outside the stochastic region. As the density rises, the maximum of the ionization source moves inwards because of the reduced ionization activities in the edge surface layer, resulting from a drop in temperature. At densities beyond rollover, most of the recycling neutrals pass through the edge surface layer, penetrating into the stochastic region and being ionized there. Now, the particles have a much longer parallel path to the targets. Note that the net frictional momentum loss is an integration of the parallel flows over the channel lengths [8]. Equivalently, the relationship between the inward shift of the ionization source and the drop of the recycling flux can also be understood as follows. In comparison to the edge surface layer, the stochastic region has much longer connection lengths (see figure 2) and has therefore a better 'confinement' for particles. A sudden inward shift of the ionization source into 
the stochastic region leads to a rise of the global particle 'confinement' time in the SOL. If the density is controlled as in the experiments (note the linear relation between $\mathrm{n}_{\mathrm{LCFS}}$ and $\bar{n}$ ), the total recycling flux (total particle source) has to drop to compensate, at least partly, the improved transport effect.

\section{Detachment transition and Marfe formation}

The detachment regime found in tokamaks is characterized by its high radiation power fraction and is, therefore, attractive not only for tokamaks but also for stellarators with regard to reducing the energy flux diverted onto targets. The radiation layer should be kept outside the confinement core to avoid a serious degradation of the global energy content. Figure 8 shows the evolution of a detachment discharge in LHD for a helical divertor configuration with $\mathrm{R}_{\mathrm{ax}}=3.65 \mathrm{~m}$. Detachment is triggered by strong gas-puffing. The transition to detachment can be identified by the abrupt increase in the total radiation measured by a bolometer. As the radiation increases, the ion saturation currents from the target probes decrease. Although the gas-puff is switched off immediately after the transition, the radiation layer moves continuously inwards into the confinement region, leading to a shrinking of the confinement region. This has been evidenced by the strong reduction of the energy content. Then, the plasma reaches a quasi steady state termed 'Serpens mode' [17], with a rotating radiation belt inside the LCFS being detected [18]. Till now, a radiation layer outside the confinement region could not yet be stabilized experimentally.

In order to get some insight into the physics underlying the detachment process, detailed numerical investigations have been started. Figure 9 shows the first simulation results, using the same mesh as shown in section 2. Except for the plasma density on the LCFS, which is used as a detachment trigger, all other input parameters, namely the SOL power, carbon sputtering coefficient and cross-field transport coefficients are kept unchanged during the density scan. 
Focusing on the detachment process, the density scan is, for a 4 MW SOL power, started from a relatively high value at which the carbon impurities radiate already $20 \%$ of the total power. With increasing density, the carbon radiation increases quickly and then saturates at a high level exceeding $90 \%$ of the SOL power. The transition to detachment is indicated by the sharp growth of the radiation and the concomitant drop in the recycling flux, as also seen in the experiments (see figure 8). The strong drop of the temperature on the LCFS results from the inward shift of the radiation layer, which will be discussed later in some detail. In spite of the strongly degraded recycling, the neutral penetration flux into the core (particle refueling) increases during and after the detachment transition because of the strongly reduced SOL screening efficiency on the recycling neutrals. This may explain the rapid increase in the plasma density observed in the detachment experiments shown in figure 8.

The simulations show that the radiation distribution pattern correlates closely with the local island structures while the radiation layer moves inwards during detachment. The EMC3 code solves stationary fluid equations and can therefore not provide any temporal information in real time space. However, the intermediate results between iterations can still give some indications on the dynamics of the radiation layer. In the simulation process, as the density passes through the shadowed region shown in figure 9, the radiation distribution changes its features from iteration to iteration. Usually, several thin, strongly localized radiation belts appear in the SOL domain. The number and the poloidal locations of the belts change while the belts move inwards across different island chains in the edge surface region. For the density steps selected, no converged solution can be found until the radiation layer touches the 10/6 island chains. The first converged radiation distribution after the detachment transition is shown in figure 10 . Comparing with the connection length contour, one find immediately a clear correlation between 
the radiation pattern and the structure of the 10/6 island chain. Radiation prefers the remnant islands. This is confirmed by further increasing the density. Now, the radiation layer jumps to the 10/7 island chain, without finding a converged solution in between.

In a detached plasma, the radiation distribution depends on local energy transport. Since the local magnetic field structure enters directly the local energy transport, it is not surprising to see a fully dynamic, multifaceted radiation pattern in a SOL of complex magnetic structure like in LHD. Indeed, the dynamics of the radiation layer on the way to the core has been indicated by the oscillation of the bolometer signals shown in figure 8. It is, however, not very clear why the remnant islands attract radiation, as found in the 3D simulations. This is certainly related to the absence of the parallel energy flux into the magnetically-isolated island core. A more detailed physics exploration is left for the future.

\section{Summary}

The SOL of the helical divertor configurations in LHD is formed by overlapping of magnetic islands, resulting from the large shear at the edge. Poincare plots show irregularities of the open field lines and indicates that the field is stochastic. On the other hand, connection length distributions show a strong correlation of the field lines, from which all the low-order island chains expected within the given l-range are indeed identified. Much clearer island contours emerge in the fluid pictures of plasma distributions calculated by the EMC3/EIRENE code, which takes the real field geometry of the SOL into account. It is found that the spatial distribution of plasma parameters correlates closely with the basic island structures of low-order resonances, as expected from regular islands. Island flattening effects are clearly seen in both simulations and Thomson scattering measurements, especially in electron temperature profiles. Opposite parallel plasma flows reside on adjacent island fans and are modulated by the island 
chains. The island chains of different resonances have poloidally shifted phase distributions. Thus, counter flow channels in neighbouring island chains can approach to each other at appropriate poloidal locations. If this happens, viscous transport causes significant momentum losses for the ions flowing in the neighbouring island chains, accounting for the absence of a high recycling regime. Both simulations and experiments show a linear increase of the divertor flux with increasing the plasma density up to a rollover point. Around the rollover point, impurity radiations (mainly from carbon) begin to increase nonlinearly and the 3D code shows an inward shift of the ionization front from the edge surface layer into the stochastic region. Closely following the rollover of the divertor flux, the plasma goes into detachment in the sense that the carbon radiation jumps to a high level. Over 90\% radiation fraction is predicted by the 3D code. After detachment transition, multi-, Marfe-like radiating belts are initially formed in the periphery of the SOL and then move inwards, with the form and phase changing and correlating with the island chains. Simulations indicate that the impurity radiation prefers the remnant island cores. Converged numerical solutions are obtained when the radiation layer passes through the 10/6 and 10/7 island chains where small closed island cores exist. However, a stable radiation layer could not yet be established inside the SOL so far. The reasons are presently unknown.

\section{Acknowledgements}

This work was financially supported by National Institute for Fusion Science, the budget code NIFS06ULPP0016.

\section{References}


[1] Ohyabu N. et al 1994 Nucl. Fusion 34387

[2] Grigull P. et al 2001 Plasma Phys. Control. Fusion 43 A175

[3] Renner H. et al 2000 Nucl. Fusion 401083

[4] Chirikov B. V. 1960 Phys. Rep. 52265

[5] Rosenbluth M. N. et al 1966 Nucl. Fusion 6297

[6] Feng Y. et al 1999 J. Nucl. Mater. 266-269 812

[7] Reiter D. et al 2005 P. 2005 Fusion Sci. Technol. 47 172-86

[8] Feng Y. et al 2006 Nucl. Fusion, 46807

[9] Rozhansky V.A. et al 2001 Nucl. Fusion 41387

[10] Rozhansky V.A. 2004 Plasma Phys. Control. Fusion 46 A1

[11] Feng Y. et al 2004 Contrib. Plasma Phys. 4457

[12] Feng Y. et al 2005 Phys. Plasmas 12052505

[13] Kobayashi M. et al 2007 J. Nucl. Mater. 363-365 294

[14] Peterson B. J. et al 2006 Plasma and Fusion Research 145

[15] Borrass K. and Janeschitz G. 1994 Nucl. Fusion, 341203

[16] Stangeby P. C. 1993 Nucl. Fusion, 331695

[17] Miyazawa J. et al 2006 Nucl. Fusion, 46532

[18] Miyazawa J. et al 2006 Plasma and Fusion Research 1026 


\section{Figure captions}

Figure 1. a) Cross-section of a subset of the computational mesh used in the simulations and b) the interface between the two mesh sets

Figure 2. Connection length contours. Top: in real geometry, bottom: in mesh coordinates with an overlying Poincare plot. Dashed lines are introduced only to guide the eye. The island chains with mode number lower than 5 become evident from correlations at different poloidal cross sections.

Figure 3. EMC3/EIRENE simulation results. Top: Te-contour, bottom: ion parallel flow distribution. Poincare plots are overlying.

Figure 4. Sketch of plasma transport processes inside an island.

Figure 5. Comparison of temperature profiles between Thomson scattering measurements (star symbols) and simulations (solid lines). The dashed lines indicate the boundaries of the simulation domain. The gradients of the measured $T_{e}$ in the inner region are indicated by the dot-dashed lines.

Figure 6. Absence of a high recycling regime and density-independence of cross-field transport. 
Figure 7. Inward shift of the ionization front with increasing plasma density around the rollover point.

Figure 8. Typical evolution of a detachment discharge in the helical divertor configuration of LHD.

Figure 9. Detachment transition process predicted by the EMC3/EIRENE code.

Figure 10. Radiation prefers remnant islands. 

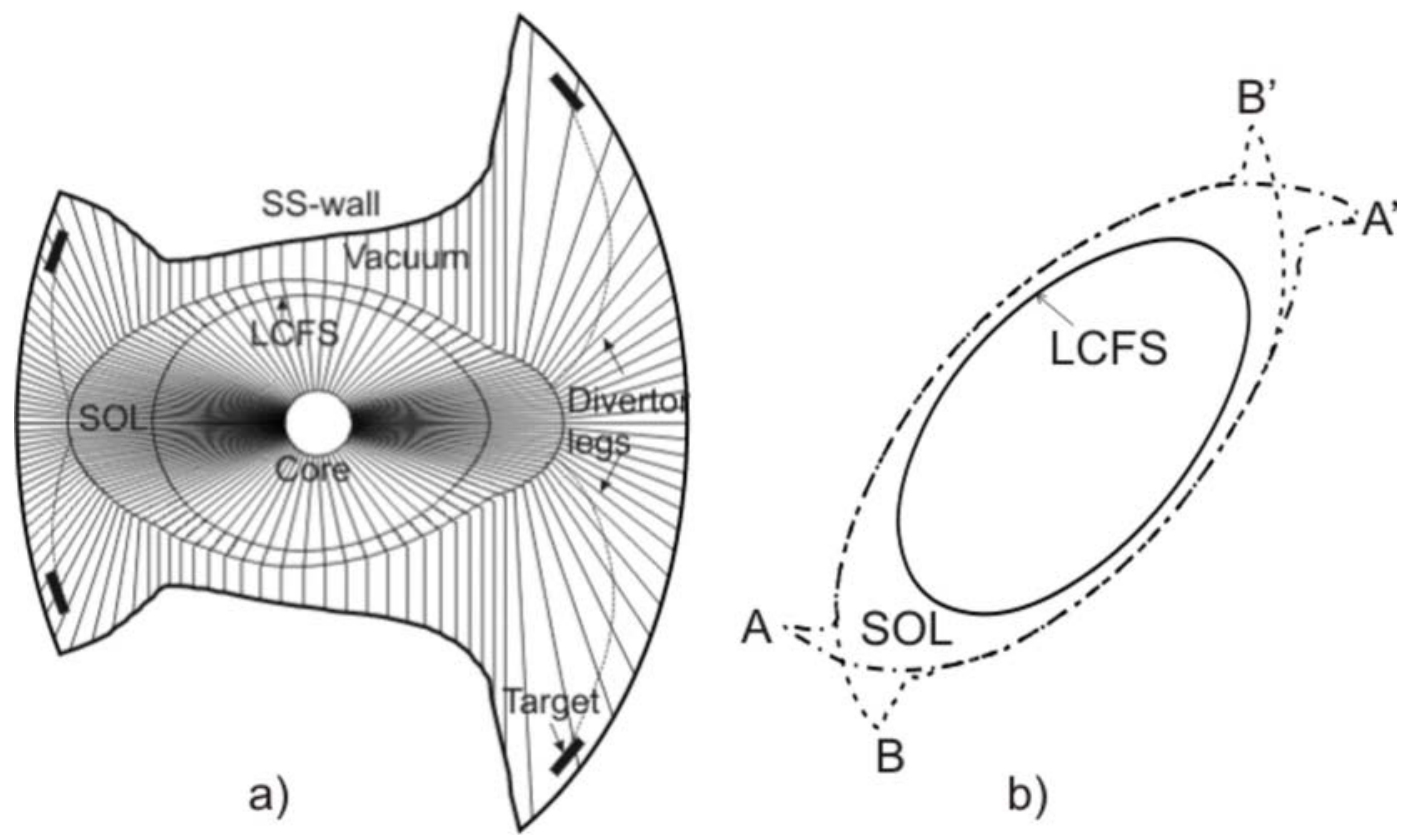

Figure 1 


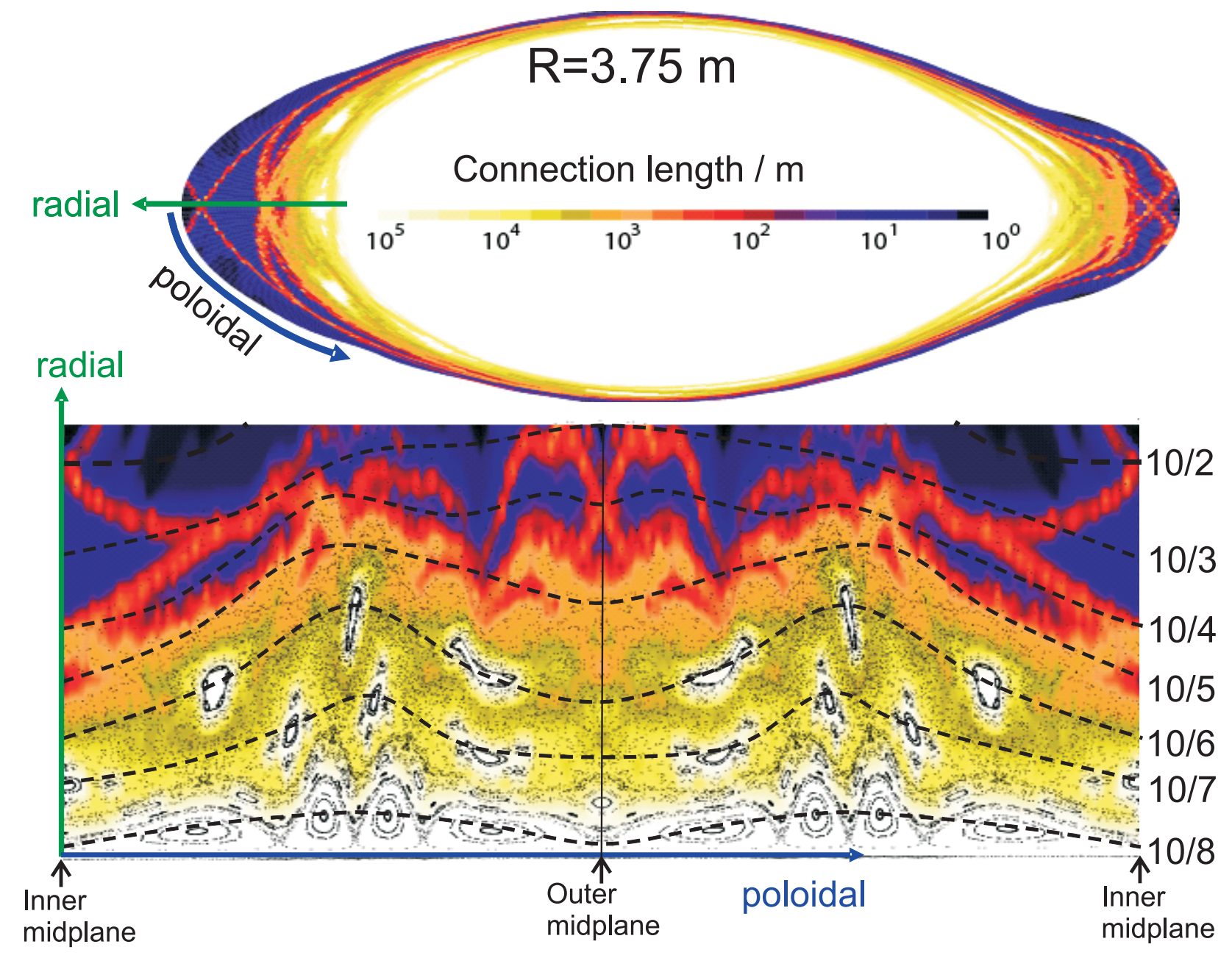

Figure 2 

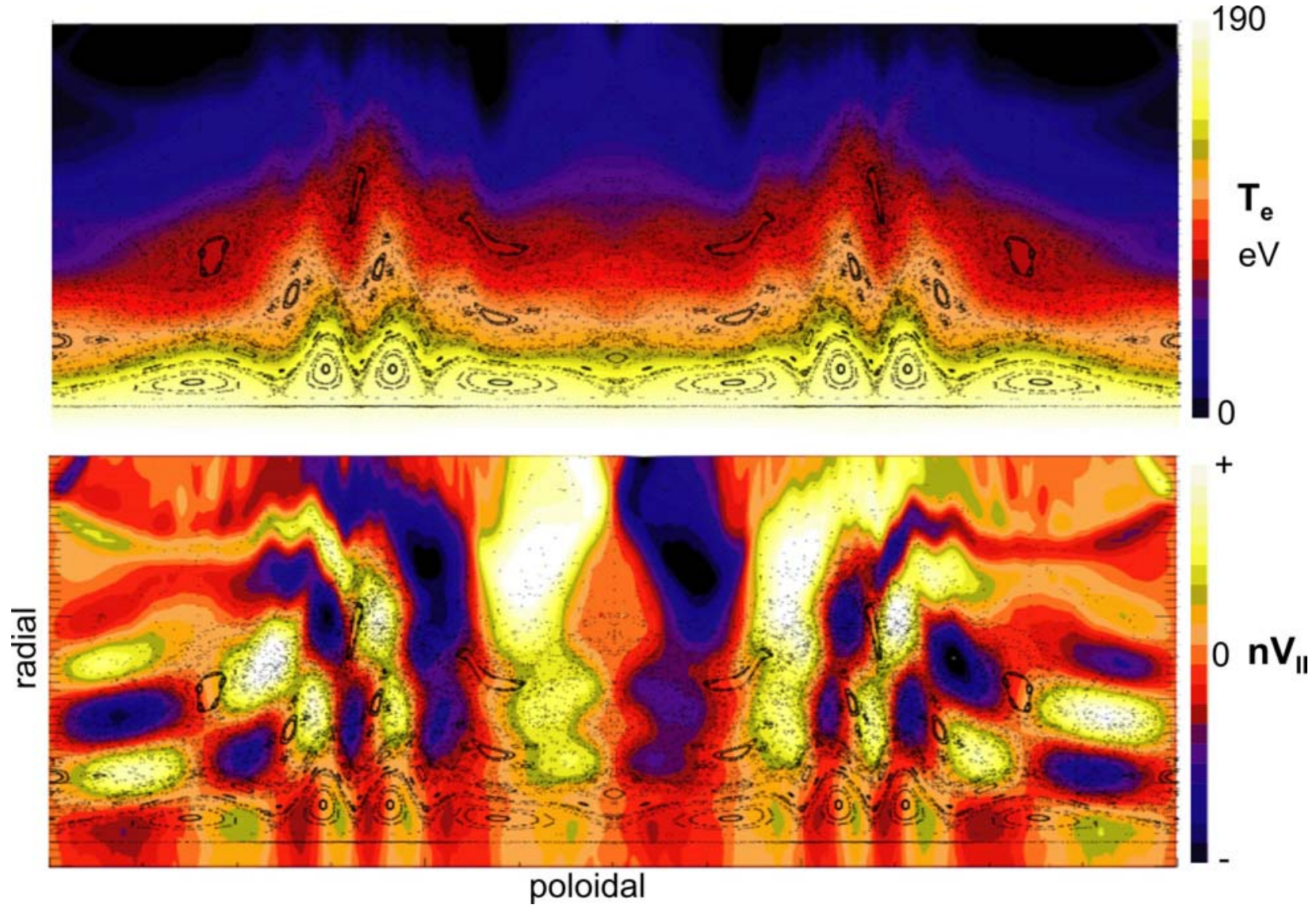

Figure 3 


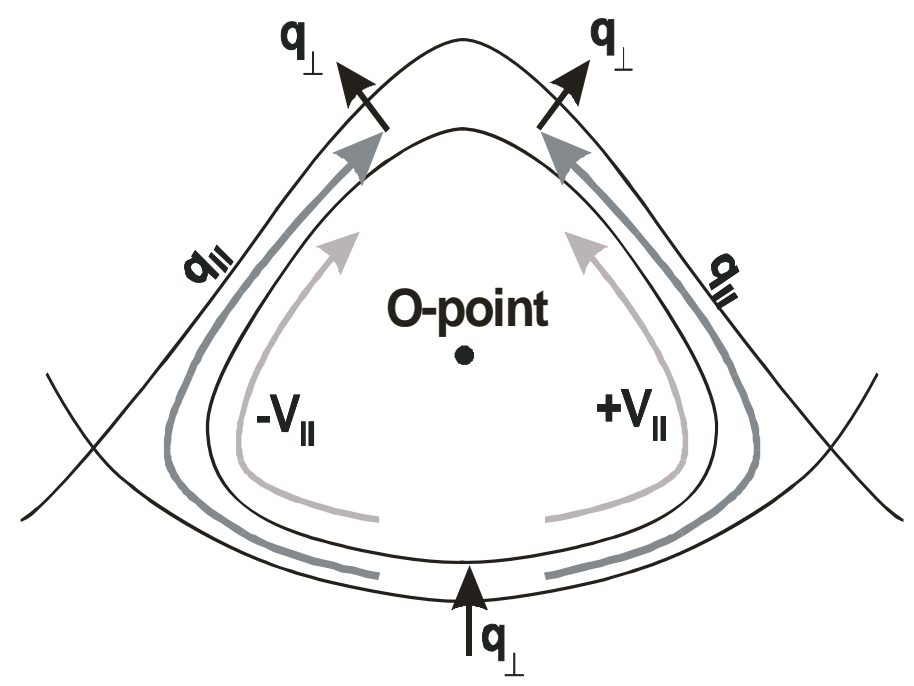

Figure 4 


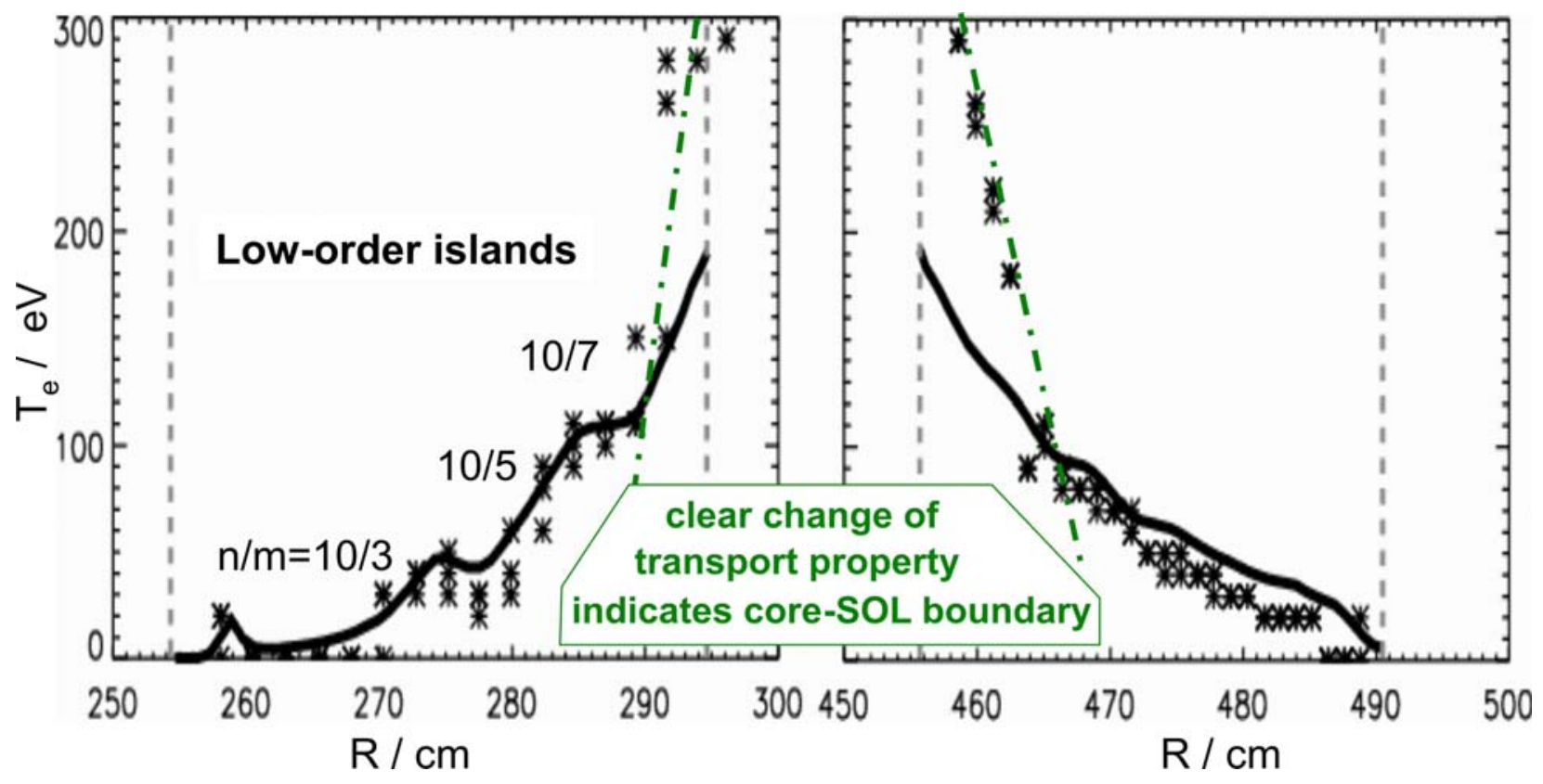

Figure 5 


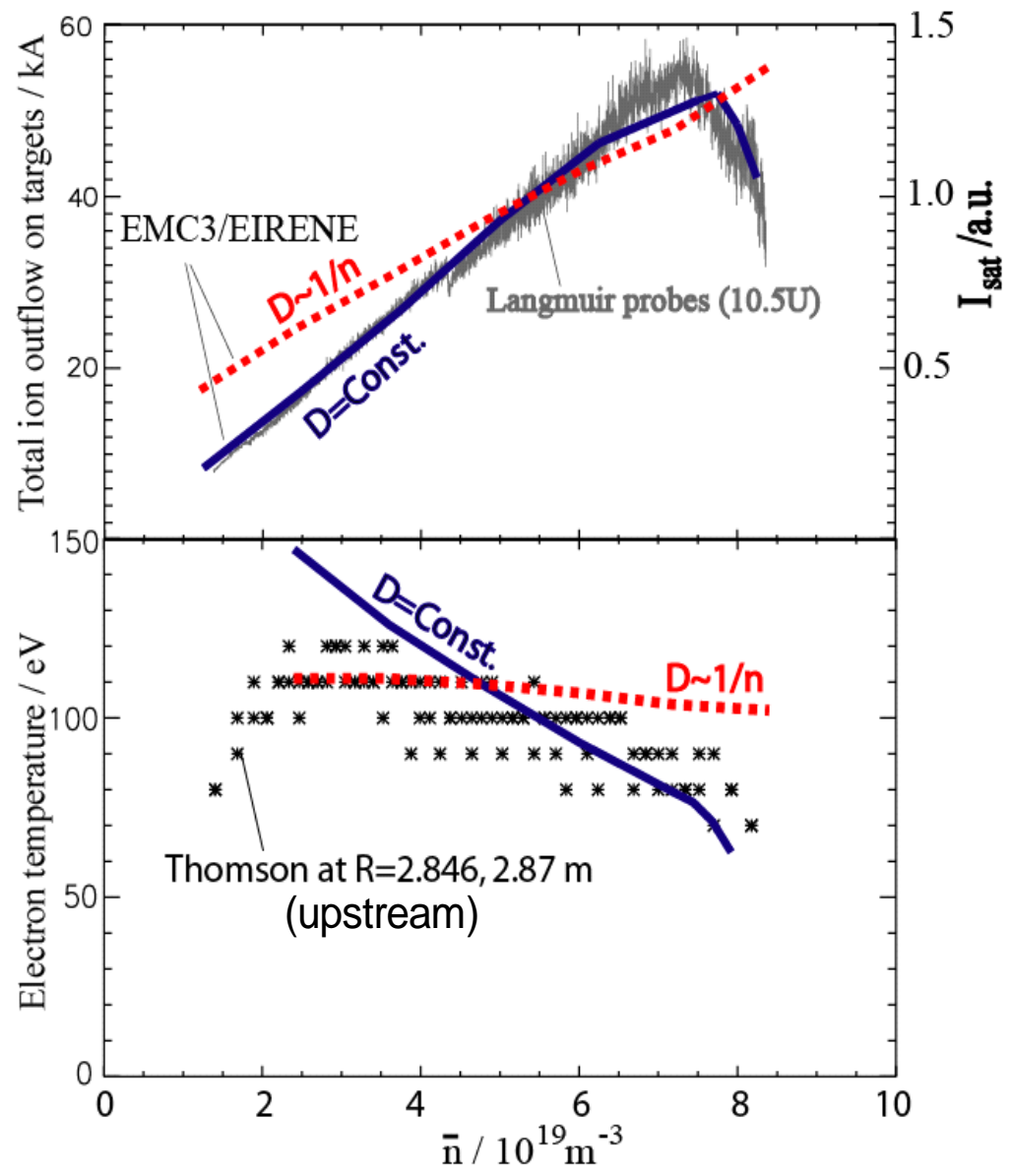

Figure 6 


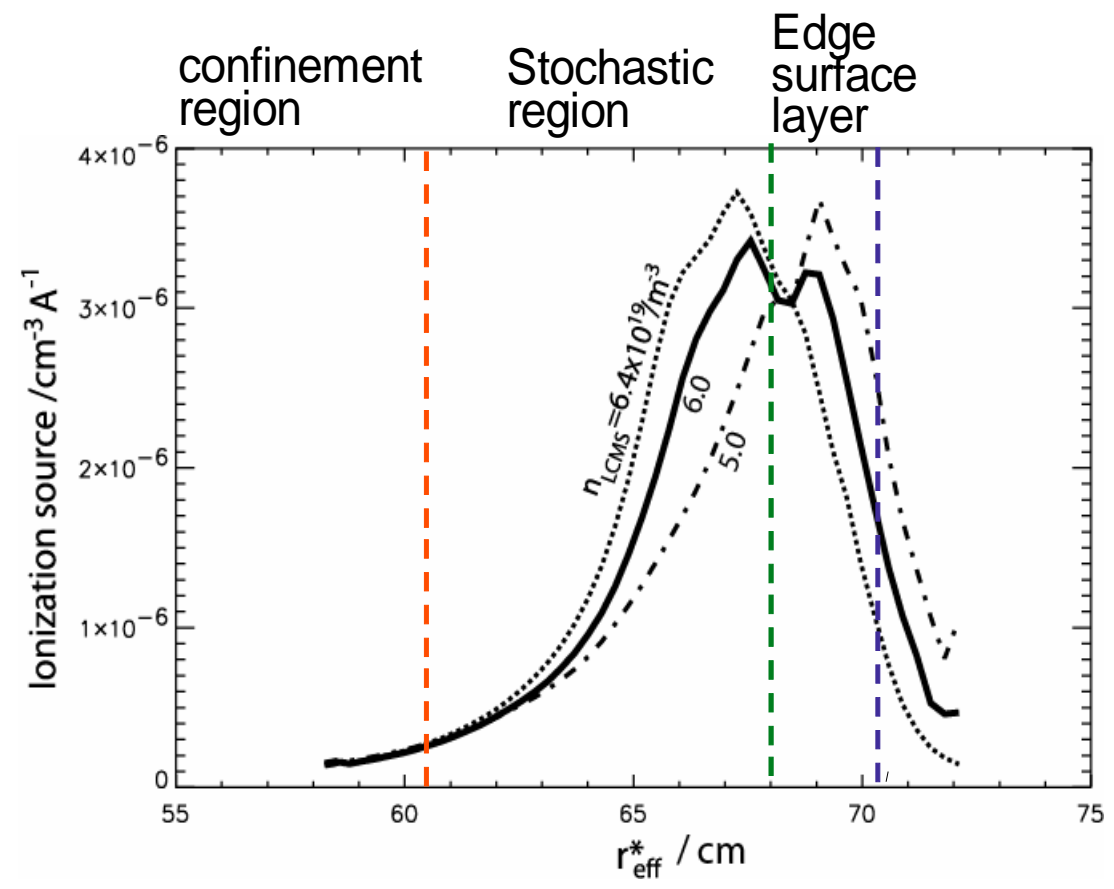

Figure 7 


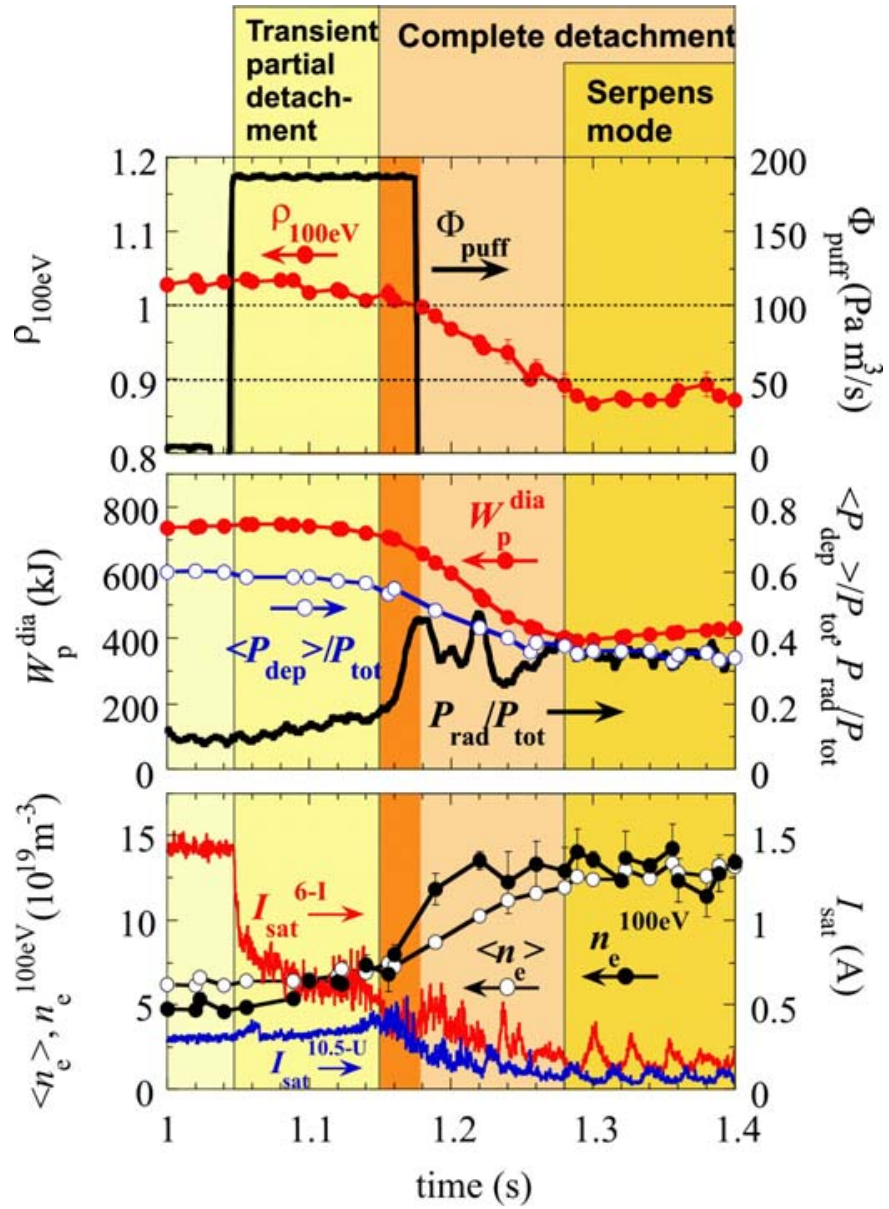

Figure 8 


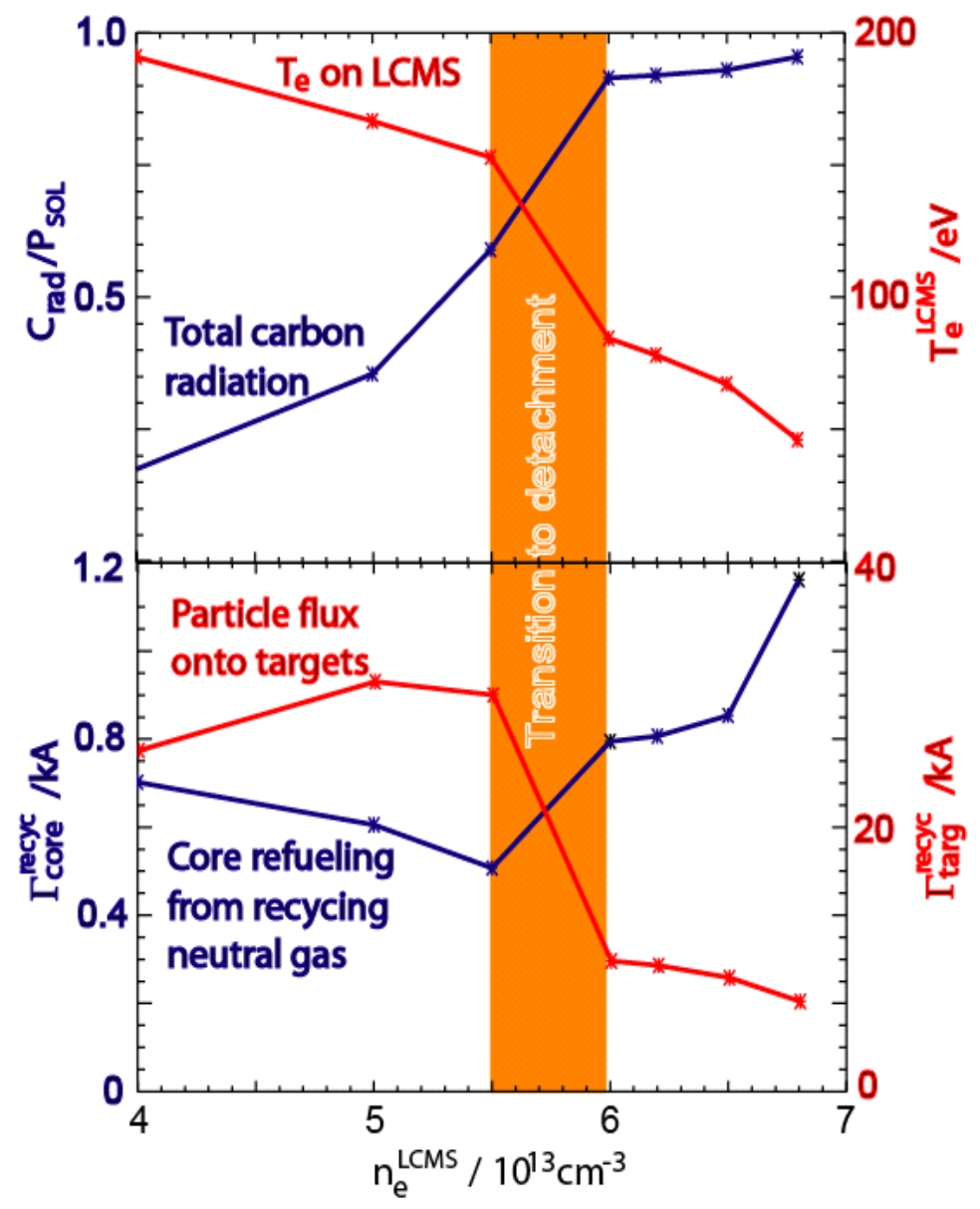

Figure 9 


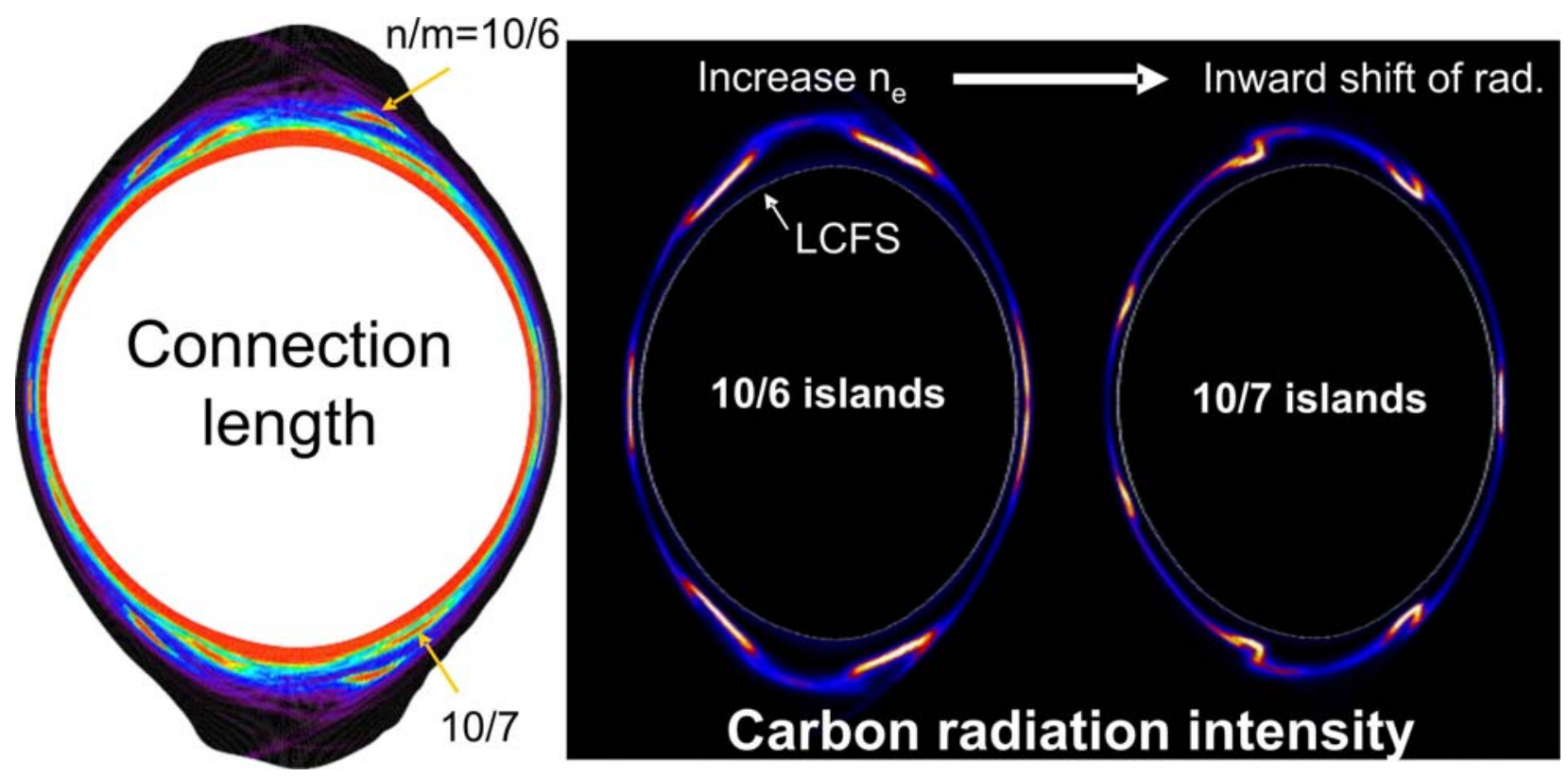

Figure 10 